\title{
Recent lattice QCD results at non-zero baryon densities
}

\author{
Jana N. Guenther* \\ Department of Physics, University of Regensburg, Universitätsstr. 31, D-93053 Regensburg, \\ Germany \\ E-mail: Jana.Guenther@t-online.de
}

\section{Rene Bellwied}

Department of Physics, University of Houston, Houston, TX 77204, USA

\section{Szabolcs Borsanyi}

Department of Physics, University of Wuppertal, Gaussstr. 20, D-42119 Wuppertal, Germany

\section{Zoltan Fodor}

Department of Physics, University of Wuppertal, Gaussstr. 20, D-42119 Wuppertal, Germany Inst. for Theoretical Physics, Eötvös University

Jülich Supercomputing Centre, Forschungszentrum Jülich, D-52425 Jülich, Germany

\section{Sandor D. Katz}

Inst. for Theoretical Physics, Eötvös University

MTA-ELTE “Lendület” Lattice Gauge Theory Research Group, Budapest, Hungary

\section{Attila Pasztor}

Department of Physics, University of Wuppertal, Gaussstr. 20, D-42119 Wuppertal, Germany

\section{Claudia Ratti}

Department of Physics, University of Houston, Houston, TX 77204, USA

\section{Kalman K. Szabo}

Department of Physics, Wuppertal University, Gaussstr. 20, D-42119 Wuppertal, Germany Jülich Supercomputing Centre, Forschungszentrum Jülich, D-52425 Jülich, Germany

\begin{abstract}
At zero baryon density lattice QCD is an established tool, that provides precise theoretical results. Calculations at non zero densities however require new techniques to deal with the sign problem. In this talk I will review the recent progress that is made in lattice QCD to investigate QCD at non-vanishing baryon chemical potential.
\end{abstract}

Critical Point and Onset of Deconfinement - CPOD2017

7-11 August, 2017

The Wang Center, Stony Brook University, Stony Brook, NY

${ }^{*}$ Speaker. 


\section{Introduction}

To analyse the quark gluon plasma that is created in heavy ion collision experiments at the LHC or RHIC a theoretical understanding of the deconfinement region of QCD is needed. Lattice QCD is a good tool to study QCD since this area can not be accessed perturbatively. At the moment direct simulations that are continuum extrapolated and at physical quark masses are restricted to vanishing or imaginary chemical potential. On the other hand the collisions especially at RHIC take place away from the axis of zero $\mu_{B}$ [1]. Therefore information in that region are needed. Even though it is not possible to do direct lattice simulations, it is possible to extrapolate observables from zero or imaginary chemical potential. This method is called analytical continuation. The analytical continuation from imaginary potential is by now well established (see for example $[2,3,4,5]$ ).

\section{The crossover temperature}

A first step for the investigation of the phase digram is the determination of the transition temperature. Since at $\mu_{B}=0$ the transition is a crossover [6] it also has to be a crossover for small values of the chemical potential. Our results for this regime that were presented in $[7,8]$ are shown in figure 1. The blue shade in the background illustrates that the crossover temperature can vary with different observables. The presented curve was gained from the chiral condensate, defined as given in [6]

$$
\langle\bar{\psi} \psi\rangle=\frac{T}{V} \frac{\partial \ln Z}{\partial m_{q}} .
$$

The renormalization was done by subtraction of lattice simulations as zero temperature:

$$
\langle\bar{\psi} \psi\rangle^{r}=-(\langle\bar{\psi} \psi\rangle(T, \beta)-\langle\bar{\psi} \psi\rangle(0, \beta)) \frac{m_{l}}{m_{\pi}^{4}}
$$

The continuum limes was calculated from simulations on lattices with sizes $40^{3} \times 10,48^{3} \times 12$ and $64^{3} \times 16$. To match the conditions in heavy ion experiments our simulations are done at the strangeness neutral point $\left\langle n_{S}\right\rangle=0$.

\section{The equation of states}

The next step in our investigation of the phase diagram is the determination of the equation of state $[9,10]$. In [11] the coefficients $c_{2}$ to $c_{6}$ were presented on $N_{t}=6$ and $N_{t}=8$ lattices. $c_{2}$ was calculated in the continuum in [12]. The most recent results on the equation of state obtained from the Taylor expansion method were presented in [13].

An important contribution for the systematic error of our results comes from the fit in the $\hat{\mu}_{B}$ direction, that is used to determine the Taylor coefficients of the pressure at $\mu_{B}=$, as well as for the extrapolation to real chemical potentials. We use the ansatz for the pressure

$$
\frac{P}{T^{4}}=p_{0}+p_{2} \frac{\mu_{B}^{2}}{T^{2}}+p_{4} \frac{\mu_{B}^{4}}{T^{4}}+p_{6} \frac{\mu_{B}^{6}}{T^{6}}+\ldots
$$

However we have no information about the higher order terms $p_{8}, \ldots$ One option to account for this terms is the use of different fit functions as done in our original analysis in $[9,10]$. However to 


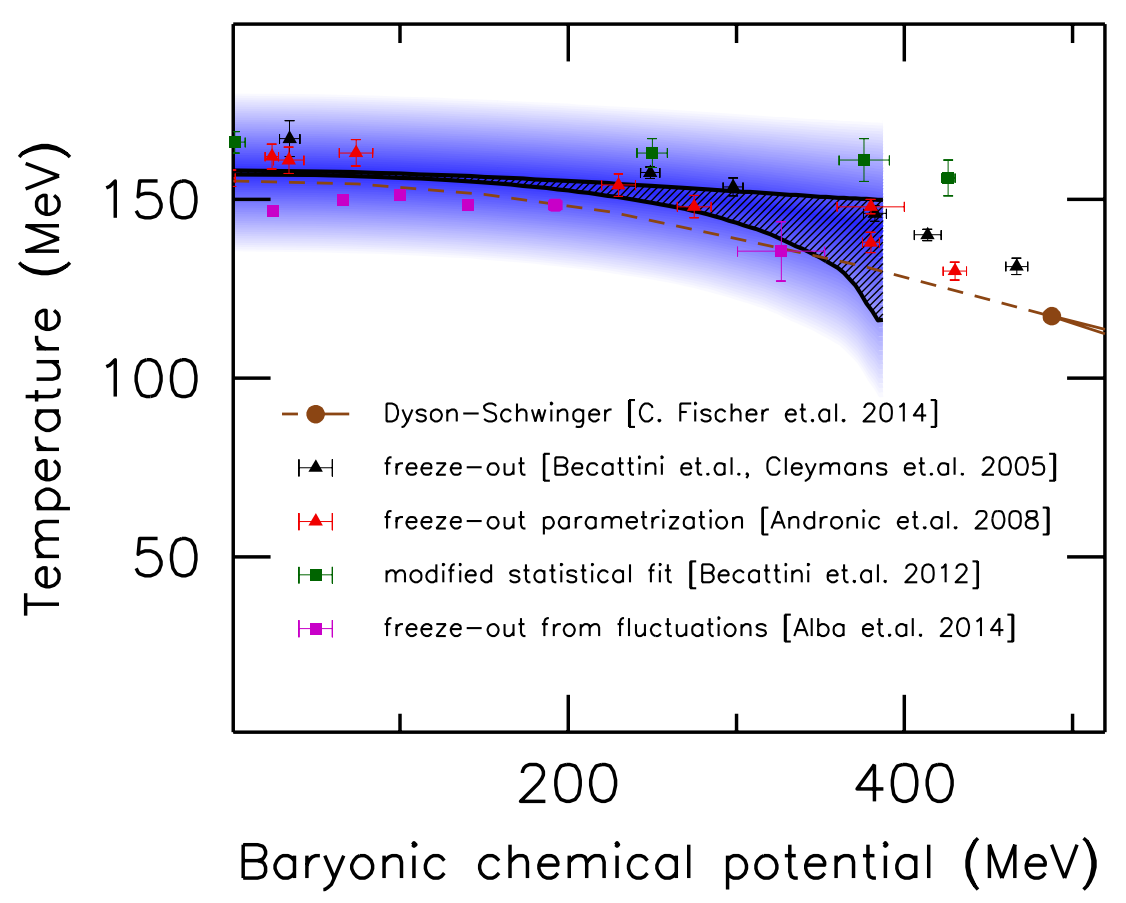

Figure 1: The extrapolation to finite chemical potential.

avoid a bias when choosing the functions we use a slightly different approach. We implement the condition

$$
\frac{\partial^{8}}{\partial \hat{\mu}_{B}^{8}}\left(\frac{P}{T^{4}}\right) \lesssim \frac{\partial^{4}}{\partial \hat{\mu}_{B}^{4}}\left(\frac{P}{T^{4}}\right)
$$

by using a fit function of the form

$$
f\left(\hat{\mu}_{B}\right)=a+b \hat{\mu}_{B}^{2}+c \hat{\mu}_{B}^{4}+\frac{b \varepsilon}{840} \hat{\mu}_{B}^{6}
$$

with

$$
\hat{\mu}_{i}=\frac{\mu_{i}}{T} .
$$

In this ansatz $a \propto p_{2}, b \propto p_{4} \ldots$ We chose 500 different values for $\varepsilon$ in equation 3.3 from a normal distribution and weight of the result is determined by the AIC information criteria [14]. The results for the coefficients $p_{2}, p_{4}$ and $p_{6}$ can be seen in figure 2 .

Besides the Taylor coefficients we compute the equation of state. In heavy ion collisions if one neglects dissipative effects, the quark gluon plasma created in the collision expands with a fixed baryon number and without generation of entropy. Therefore the ratio $\frac{S}{N_{B}}$ is constant. The medium cools down along isentropic trajectories (where $\frac{S}{N_{B}}$ is constant) in the $T-\mu_{B}$-plane. Therefore we 
compute the equation of state along this trajectories. In figure 3 we show six trajectories that were matched to the beem energies of RHIC determined in [15]. The solid lines are predictions of the HRG model that we use to continue our trajectories at lower temperatures. As it can be seen from the errors the trajectory with $\frac{S}{N_{B}}=30$ corresponding to a beam energy of $14.5 \mathrm{GeV}$ is at the end of how far we can extrapolate in $\mu_{B}$. As the error there is relatively large we compute the equation of state along the trajectories with $\frac{S}{N_{B}}=51$ and $\frac{S}{N_{B}}=420$. Figure 4 shows the pressure and the trace anomaly along the isentropic trajectories.
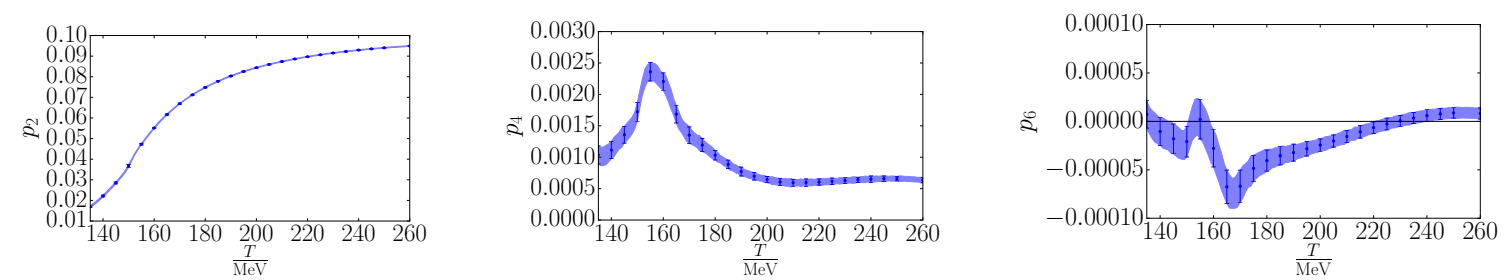

Figure 2: The Taylor coefficients of the pressure obtained from a fit function of the form given in equation (3.3).

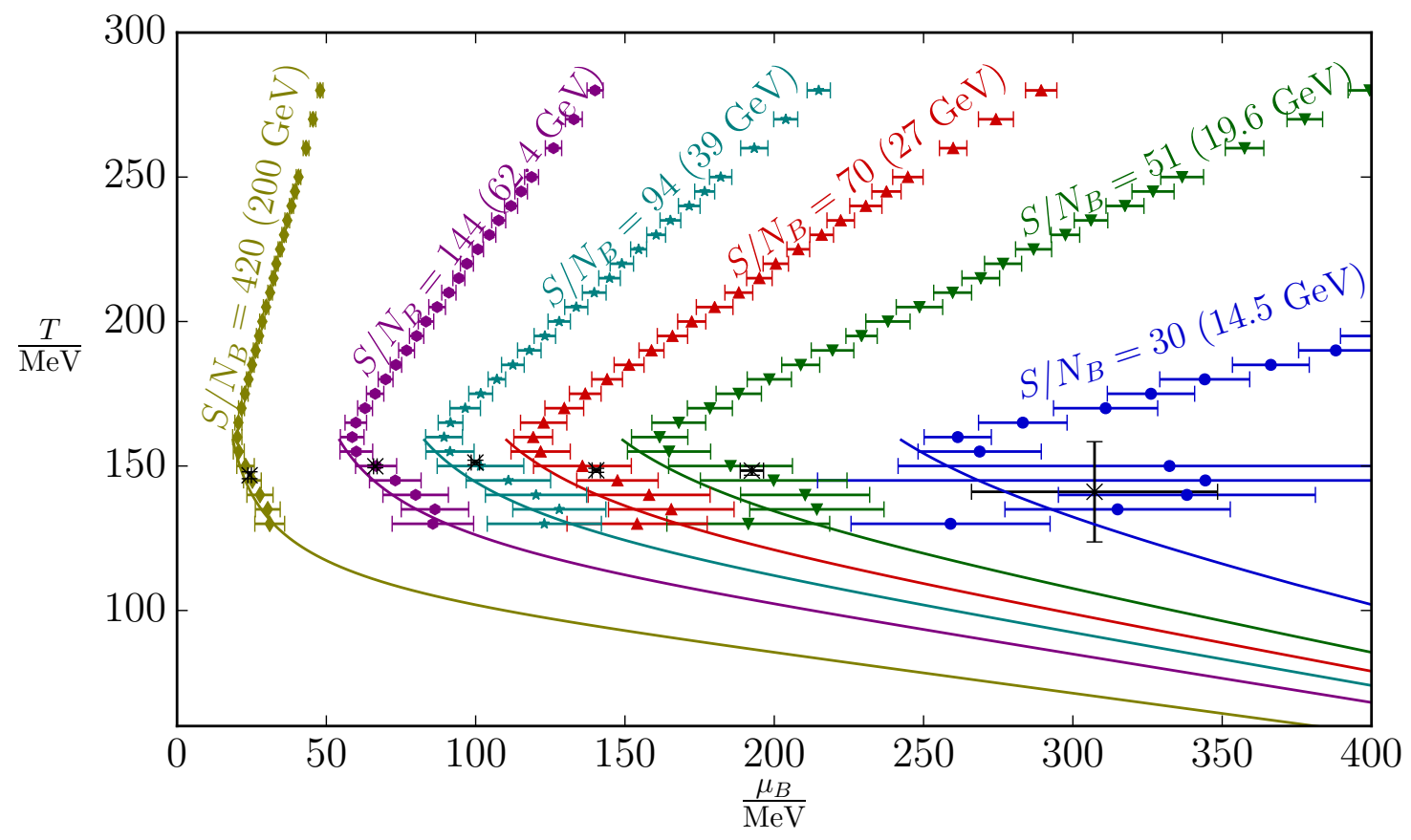

Figure 3: The trajectories of constant $\frac{S}{N_{B}}$ matched to the beam energies of RHIC as determined in [15].

\section{Fluctuations}

Fluctuations can be measured on the particle distributions in heavy ion collisions (see for example [16]). A comparison between the experimental measurements and the theoretical calcu- 


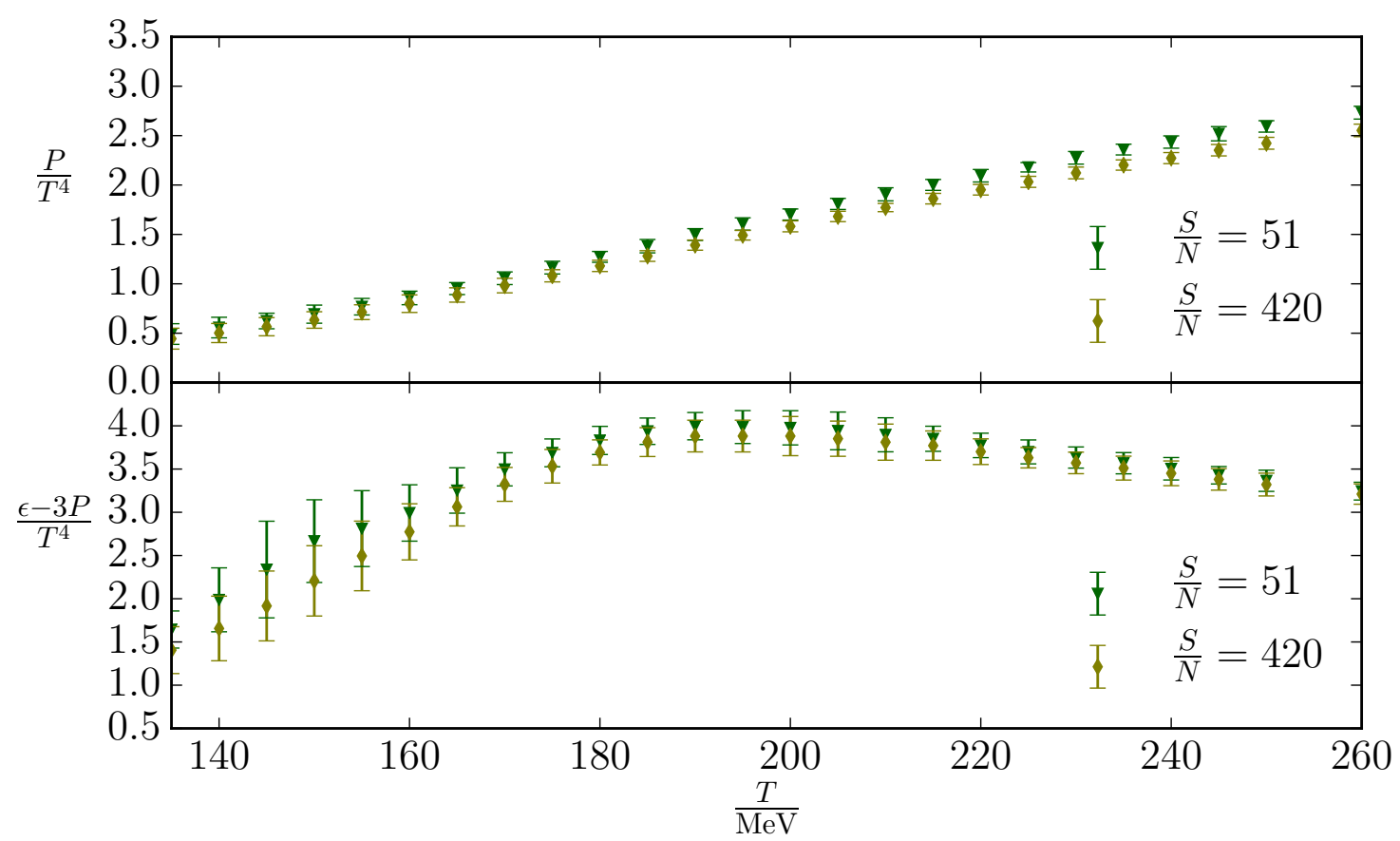

Figure 4: The pressure and the trance anomaly along trajectories of constant $\frac{S}{N}$.

lations allows then for the determination of the order of the transition. A similar analysis form simulations at imaginary chemical potential was done in [5], that we will improve upon by presenting calculations to higher orders in $\mu_{B}$. Results on the same observables were also studied by the Taylor expansion method and published in [13]. In contrast to our analysis for the transition temperature and the equation of state (section 2 and 3) the simulations for the fluctuations are done on lattice of sice $48^{3} \times 12$ with $\mu_{S}=\mu_{Q}=0$. We present our analysis in three steps. We start with $\chi_{2}^{B}, \chi_{4}^{B}, \chi_{6}^{B}$ and $\chi_{8}^{B}$ where we use the notation

$$
\chi_{i, j, k}^{B, Q, S}=\frac{\partial^{i+j+k}\left(p / T^{4}\right)}{\left(\partial \hat{\mu}_{B}\right)^{i}\left(\partial \hat{\mu}_{Q}\right)^{j}\left(\partial \hat{\mu}_{S}\right)^{k}} .
$$

First we do the analysis for each temperature separately (section 4.1). Afterwards we use the information that the results are expected to lie on a smooth curve, by introducing a spline through the results (section 4.2). Finally we use the same techniques introduced for $\chi_{i}^{B}$ to calculate three different ratios of the cumulants of the baryon distribution at $\left\langle n_{S}\right\rangle=0$ and $\left\langle n_{Q}\right\rangle=0.4\left\langle n_{B}\right\rangle$ in terms of the $\chi_{i, j, k}^{B, Q, S}$ (section 4.3).

\subsection{Single Temperature}

First we analyse the data for a single temperature. For each $\mu_{B} \neq 0$ we measure $\chi_{1}^{B}, \chi_{2}^{B}, \chi_{3}^{B}$ and $\chi_{4}^{B}$, while for $\mu_{B}=0$ only $\chi_{2}^{B}$ and $\chi_{4}^{B}$ are measured since $\chi_{1}^{B}$ and $\chi_{3}^{B}$ are odd functions in $\mu_{B}$ and therefore equal to zero. 
We make the ansatz for the partition sum:

$$
\chi_{0}^{B} \hat{\mu}_{B}=c_{0}+c_{2} \hat{\mu}_{B}^{2}+c_{4} \hat{\mu}_{B}^{4}+c_{6} \hat{\mu}_{B}^{6}+c_{8} \hat{\mu}_{B}^{8} .
$$

From this we can calculate the derivatives that we can measure on the lattice:

$$
\begin{aligned}
& \chi_{1}^{B} \hat{\mu}_{B}=2 c_{2} \hat{\mu}_{B}+4 c_{4} \hat{\mu}_{B}^{3}+6 c_{6} \hat{\mu}_{B}^{5}+8 c_{8} \hat{\mu}_{B}^{7} \\
& \chi_{2}^{B} \hat{\mu}_{B}=2 c_{2}+12 c_{4} \hat{\mu}_{B}^{2}+30 c_{6} \hat{\mu}_{B}^{4}+56 c_{8} \hat{\mu}_{B}^{6} \\
& \chi_{3}^{B} \hat{\mu}_{B}=24 c_{4} \hat{\mu}_{B}+120 c_{6} \hat{\mu}_{B}^{3}+336 c_{8} \hat{\mu}_{B}^{5} \\
& \chi_{4}^{B} \hat{\mu}_{B}=24 c_{4}+360 c_{6} \hat{\mu}_{B}^{2}+1680 c_{8} \hat{\mu}_{B}^{4} .
\end{aligned}
$$

In a first step we determine the coefficients $c_{2}, c_{4}$ and $c_{6}$ from a correlated fit to the data, while $c_{8}$ is set to zero. The results are shown as blue points in figure 5. This ansatz does not account for the systematic uncertainties that arise from higher order contributions in $\frac{\mu_{B}}{T}$. To obtain a first estimate for this uncertainties we include $c_{8}$ in our fit and check for changes in $c_{2}$ to $c_{6}$, which are shown in figure 6. While the results for $\chi_{2}^{B}$ and $\chi_{4}^{B}$ remain mostly unchanged, the changes for $\chi_{6}^{B}$ after the inclusion of $\chi_{8}^{B}$ are significant. This stresses that a careful investigation of the influences of higher orders are necessary.
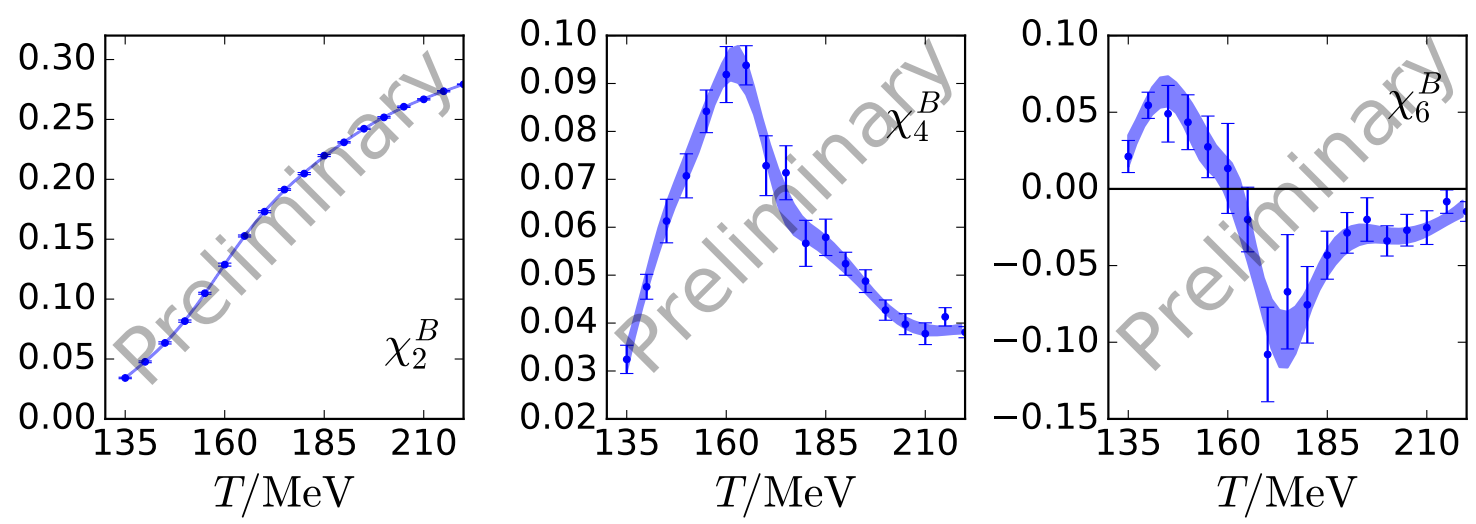

Figure 5: Preliminary results for $\chi_{2}^{B}, \chi_{4}^{B}$ and $\chi_{6}^{B}$

\subsection{Spline Fit}

We expect our results for $\chi_{i}^{B}(T)$ to lie on a smooth curve. To implement this information we fit the results with a spline. The fit parameters $c_{2}, c_{4}, c_{6}$ and $c_{8}$ now become functions of $T$ themselves. For the spline fitting procedure the choice of note points is crucial. To reduce the bias that is implemented with a specific choice we have three different modes. The first and possibley simplest method is just to have all note points evenly spaced. However at higher temperatures we expect slower changes in our curves. Also for $\mu_{B}=0$ our simulation points at high temperatures are more sparse. Therefore as a second choice we double the distance between the last three note points. As a last possibility we chose our note points randomly, with a uniform distribution. However to avoid the case of two note points that are very close to each other we only except a configuration if the 

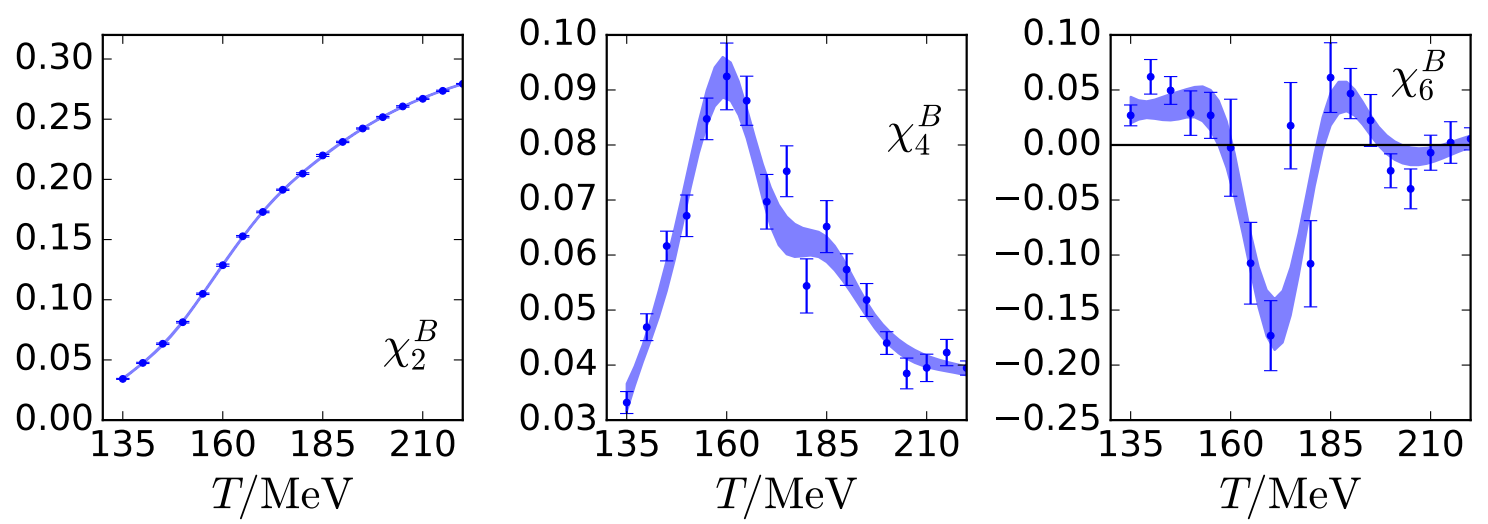

Figure 6: Preliminary results for $\chi_{2}^{B}, \chi_{4}^{B}$ and $\chi_{6}^{B}$ when $\chi_{8}^{B}$ is included in the fit.

distance between two neighbouring note points is at least $10 \mathrm{MeV}$. An illustration of this different node point configurations is shown in figure 7 . Also the number of note points is varied between six and eight. The results are shown by the blue band in figure 5 and figure 6 .

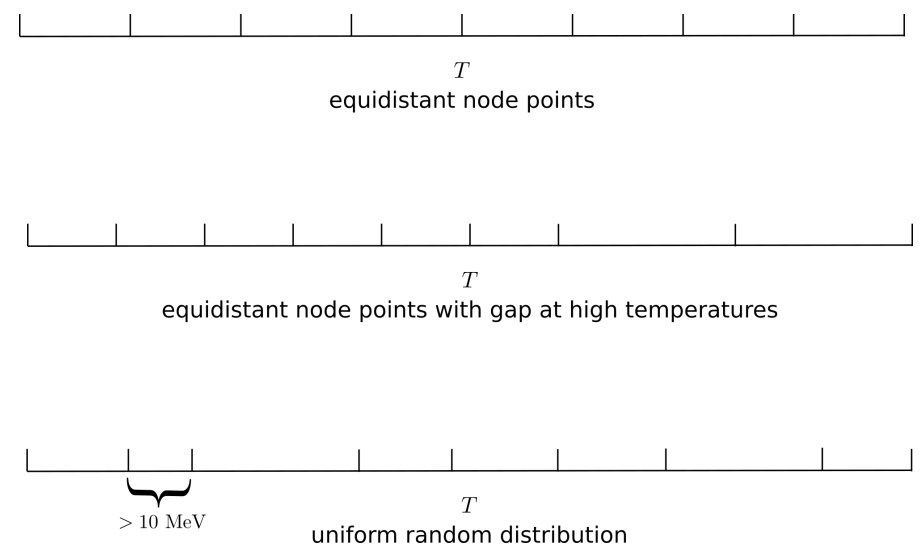

Figure 7: Different techniques for choosing the spline node points.

\subsection{Cumulants}

For a comparison with heavy ion collision experiments the cumulants of the net baryon distribution are a useful tool. The first four cumulants are the mean $M_{B}$, the variance $\sigma_{B}^{2}$, the skewness $S_{B}$ and the kurtosis $\kappa_{B}$. By forming appropriate ratios, we can cancel out explicit volume factors. However the measured distributions themselves may still depend on the volume, which one should take into account, when comparing to experiments.

Heavy ion collisions with lead or gold take place with at $\mu_{B}>0,\left\langle n_{S}\right\rangle=0$ and $\left\langle n_{Q}\right\rangle=0.4\left\langle n_{B}\right\rangle$. Since our simulations are done at $\mu_{S}=\mu_{Q}=0$ and $\mu_{B} \neq 0$ we have to do some calculations to arrive at the same observables that are measured in experiments (see for example [16]). We investigate 
three different rations of cumulants and write each as a Taylor expansion:

$$
\begin{gathered}
\frac{M_{B}}{\sigma_{B}^{2}}=\frac{\chi_{1}^{B}\left(T, \hat{\mu}_{B}\right)}{\chi_{2}^{B}\left(T, \hat{\mu}_{B}\right)}=\hat{\mu}_{B} r_{12}^{B, 1}+\hat{\mu}_{B}^{3} r_{12}^{B, 3}+\ldots \\
\frac{S_{B} \sigma_{B}^{3}}{M_{B}}=\frac{\chi_{3}^{B}\left(T, \hat{\mu}_{B}\right)}{\chi_{1}^{B}\left(T, \hat{\mu}_{B}\right)}=r_{31}^{B, 0}+\hat{\mu}_{B}^{2} r_{31}^{B, 2}+\ldots \\
\kappa_{B} \sigma_{B}^{2}=\frac{\chi_{4}^{B}\left(T, \hat{\mu}_{B}\right)}{\chi_{2}^{B}\left(T, \hat{\mu}_{B}\right)}=r_{42}^{B, 0}+\hat{\mu}_{B}^{2} r_{42}^{B, 2}+\ldots
\end{gathered}
$$

The $\mu_{B}$ dependence of the $\chi_{i}^{B}\left(T, \hat{\mu}_{B}\right)$ can be again written in terms of the Taylor expansion:

$$
\begin{aligned}
\chi_{i, j, k}^{B Q S}\left(\hat{\mu}_{B}\right) & =\chi_{i, j, k}^{B Q S}(0)+\hat{\mu}_{B}\left[\chi_{i+1, j, k}^{B Q S}(0)+q_{1} \chi_{i, j+1, k}^{B Q S}(0)+s_{1} \chi_{i, j, k+1}^{B Q S}(0)\right] \\
& +\frac{1}{2} \hat{\mu}_{B}^{2}\left[\chi_{i+2, j, k}^{B Q S}(0)+s_{1}^{2} \chi_{i, j+2, k}^{B Q S}(0)+q_{1}^{2} \chi_{i, j, k+2}^{B Q S}(0)\right. \\
& \left.+2 q_{1} s_{1} \chi_{i, j+1, k+1}^{B Q S}(0)+2 s_{1} \chi_{i+1, j+1, k}^{B Q S}(0)+2 q_{1} \chi_{i+1, j, k+1}^{B Q S}(0)\right]+\ldots
\end{aligned}
$$

with

$$
\begin{aligned}
q_{j} & =\frac{1}{j !} \frac{d^{j} \hat{\mu}_{Q}}{\left(d \hat{\mu}_{B}\right)^{j}}(0) \\
s_{j} & =\frac{1}{j !} \frac{d^{j} \hat{\mu}_{S}}{\left(d \hat{\mu}_{B}\right)^{j}}(0)
\end{aligned}
$$

We can now use the constrains $\left\langle n_{S}\right\rangle=0$ and $\left\langle n_{Q}\right\rangle=0.4\left\langle n_{B}\right\rangle$ which can be rewritten as

$$
\chi_{1}^{Q}=0.4 \chi_{1}^{B}, \quad \chi_{1}^{S}=0
$$

to determine $r_{i j}^{B, k}$ coefficients form the equations (4.7), (4.8) and (4.9). However we now need to know not only the behaviour of the $\chi_{i}^{B}$ but also of derivatives with respect to $\mu_{S}$ and $\mu_{Q}$. For now our simulations are restricted to ensembles with finite $\mu_{B}$. Therefore the $\mu_{S}$ and $\mu_{Q}$ derivatives have to be calculated directly and without the support from the fit that we used in the $\mu_{B}$ direction. We calculate various $\chi_{i, j, k}^{B, Q, S}$ with the appropriate values of $j$ and $k$ and all possible values for $i$ so that

$$
i+j+k \leq 4
$$

For each group of fluctuations with the same $j$ and $k$ we perform a fit analogous to the procedure described in the sections 4.1 and 4.2. This is sufficient to determine the first to $r_{i j}^{B, k}$ coefficients for all three observables. The results are shown in figure 8, 9 and 10. For higher order coefficients, higher order derivatives in $\mu_{S}$ and $\mu_{Q}$ are needed. The direct measurements have a rapidly increasing error with each derivative and very large statistics would be needed to improve our calculations in that manner. Another possibility would be add ensembles with finite $\mu_{S}$ and $\mu_{Q}$ and do a similar fit as for the $\mu_{B}$ direction. This approach has been used in [5]. 

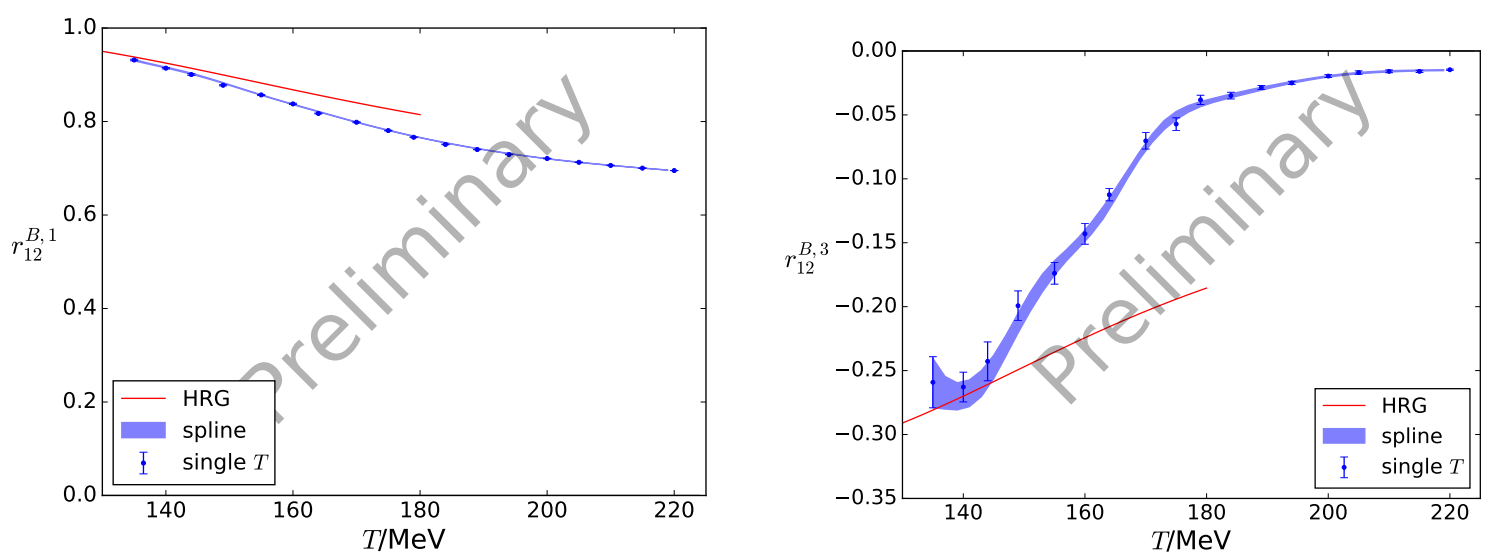

Figure 8: $\frac{M_{B}}{\sigma_{B}^{2}}=\frac{\chi_{1}^{B}\left(T, \hat{\mu}_{B}\right)}{\chi_{2}^{B}\left(T, \hat{\mu}_{B}\right)}=\hat{\mu}_{B} r_{12}^{B, 1}+\hat{\mu}_{B}^{3} r_{12}^{B, 3}+\ldots$
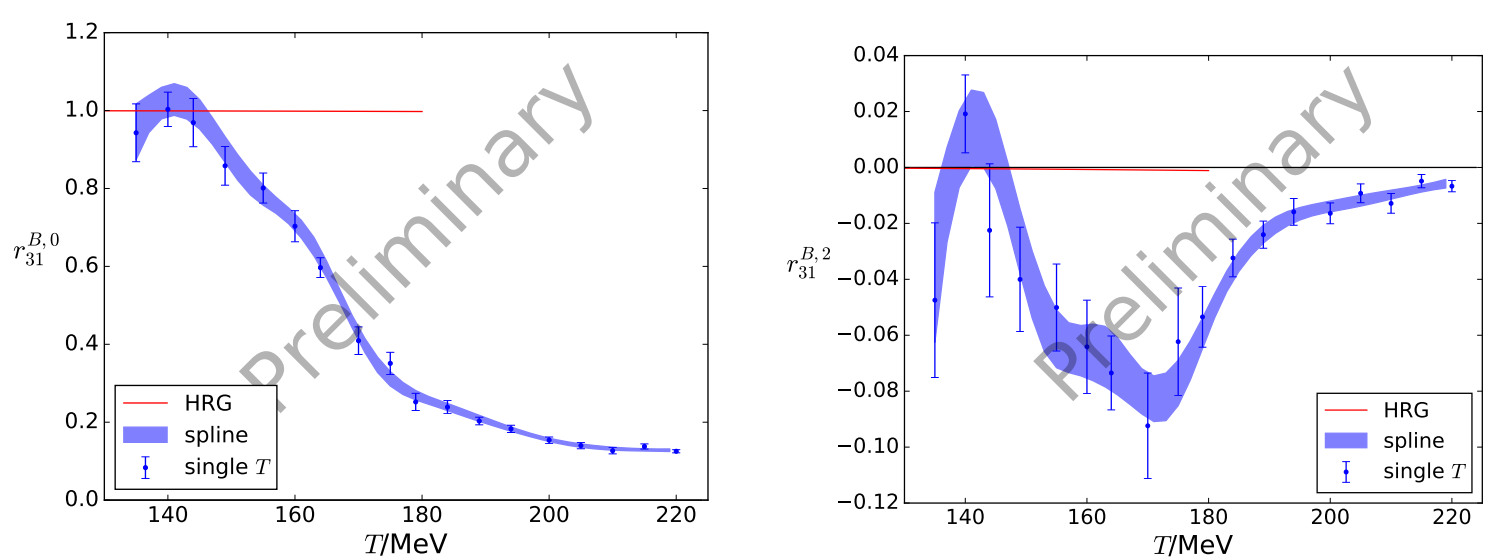

Figure 9: $\frac{S_{B} \sigma_{B}^{3}}{M_{B}}=\frac{\chi_{3}^{B}\left(T, \hat{\mu}_{B}\right)}{\chi_{1}^{B}\left(T, \hat{\mu}_{B}\right)}=r_{31}^{B, 0}+\hat{\mu}_{B}^{2} r_{31}^{B, 2}+\ldots$
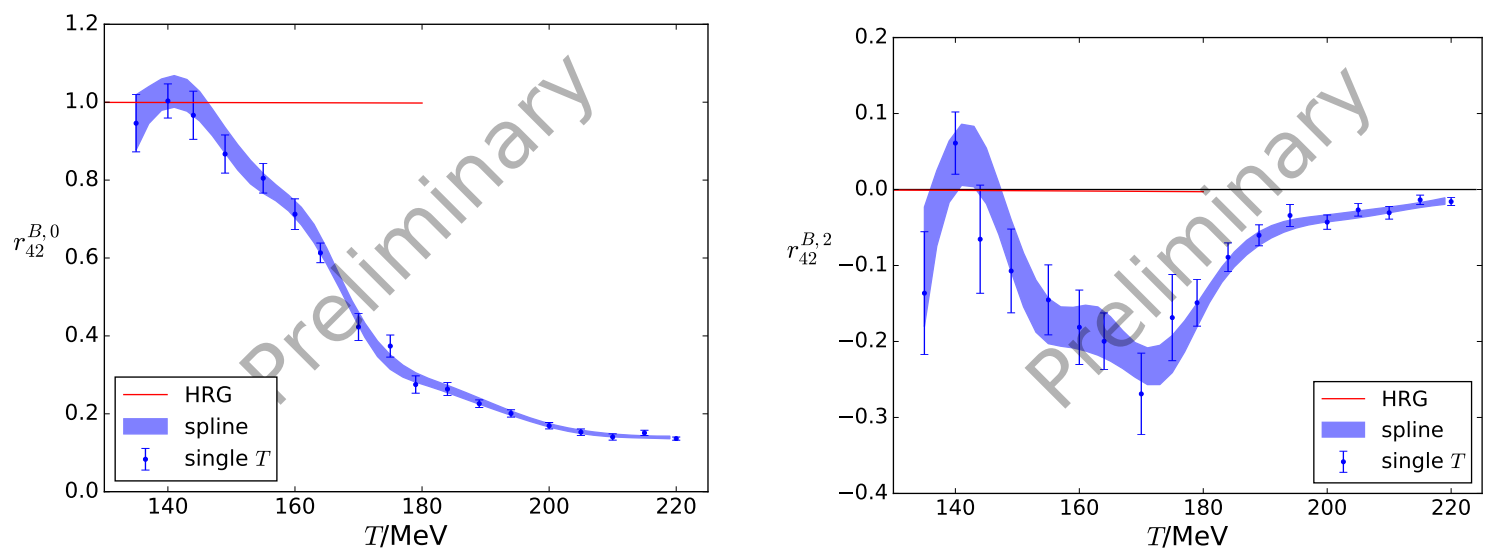

Figure 10: $\kappa_{B} \sigma_{B}^{2}=\frac{\chi_{4}^{B}\left(T, \hat{\mu}_{B}\right)}{\chi_{2}^{B}\left(T, \hat{\mu}_{B}\right)}=r_{42}^{B, 0}+\hat{\mu}_{B}^{2} r_{42}^{B, 2}+\ldots$ 


\subsection{Error Analysis}

For a reliable comparison between experimental measurements and theoretical calculations the error estimation is an important ingredient. As we present work in progress results the error estimation process is not yet finished. Our statistical error is estimated by the Jack-Knife-Method. For our systematic error there are several sources, which we have not yet completely covered. We determine our systematic error by the histogram method described in [17], where each analysis is weighted with the akaike information criteria. We include the influence of the number of points in the $\mu_{B}$ direction, by either including or ignoring the data from our highest value for $\mu_{B}$. We also try to estimate the influence of the spline node points as described in section 4.2. However a very important source for our systematic error is the influence of the higher order contributions in $\mu_{B}$ that are not included in our fit ansatz. A rough idea of this influences can be gained from the comparison of the results shown in figure 5 and figure 6 , where one order more was included. However a more detailed analysis is necessary to obtain a reliable error on our result.

\section{Acknowledgement}

This project was funded by the DFG grant SFB/TR55. This material is based upon work supported by the National Science Foundation through grant number NSF PHY-1654219 and by the U.S. Department of Energy, Office of Science, Office of Nuclear Physics, within the framework of the Beam Energy Scan Theory (BEST) Topical Collaboration. An award of computer time was provided by the INCITE program. This research used resources of the Argonne Leadership Computing Facility, which is a DOE Office of Science User Facility supported under Contract DE-AC02-06CH11357. The authors gratefully acknowledge the Gauss Centre for Supercomputing (GCS) for providing computing time for a GCS Large-Scale Project on the GCS share of the supercomputer JUQUEEN [18] at Jülich Supercomputing Centre (JSC). The authors gratefully acknowledge the use of the Maxwell Cluster and the advanced support from the Center of Advanced Computing and Data Systems at the University of Houston.

\section{References}

[1] G. Odyniec, Future of the beam energy scan program at RHIC, EPJ Web Conf. 95 (2015) 03027.

[2] P. de Forcrand and O. Philipsen, The QCD phase diagram for small densities from imaginary chemical potential, Nucl. Phys. B642 (2002) 290-306, [hep-lat/ 0205016$].$

[3] C. Bonati, M. D'Elia, M. Mariti, M. Mesiti, F. Negro and F. Sanfilippo, Curvature of the chiral pseudocritical line in QCD: Continuum extrapolated results, Phys. Rev. D92 (2015) 054503, [1507.03571].

[4] P. Cea, L. Cosmai and A. Papa, Critical line of 2+1 flavor QCD: Toward the continuum limit, Phys. Rev. D93 (2016) 014507, [1508.07599].

[5] M. D'Elia, G. Gagliardi and F. Sanfilippo, Higher order quark number fluctuations via imaginary chemical potentials in $N_{f}=2+1$ QCD, Phys. Rev. D95 (2017) 094503, [1611.08285].

[6] Wuppertal-Budapest collaboration, S. Borsanyi, Z. Fodor, C. Hoelbling, S. D. Katz, S. Krieg, C. Ratti et al., Is there still any $T_{c}$ mystery in lattice QCD? Results with physical masses in the continuum limit III, JHEP 09 (2010) 073, [1005.3508]. 
[7] R. Bellwied, S. Borsanyi, Z. Fodor, J. Guenther, S. D. Katz, C. Ratti et al., The QCD phase diagram from analytic continuation, Phys. Lett. B751 (2015) 559-564, [1507. 07510].

[8] J. Guenther, R. Bellwied, S. Borsanyi, Z. Fodor, S. D. Katz, C. Ratti et al., The curvature of the crossover line in the $(T, \mu)$-phase diagram of $Q C D, P o S$ LATTICE2015 (2016) 142.

[9] J. Guenther, R. Bellwied, S. Borsanyi, Z. Fodor, S. D. Katz, A. Pasztor et al., The QCD equation of state at finite density from analytical continuation, 1607.02493.

[10] J. Gunther, R. Bellwied, S. Borsanyi, Z. Fodor, S. D. Katz, A. Pasztor et al., The QCD equation of state at finite density from analytical continuation, EPJ Web Conf. 137 (2017) 07008, [1607.02493].

[11] BNL-BIELEFELD-CCNU collaboration, P. Hegde, The QCD equation of state to $\mathscr{O}\left(\mu_{B}^{4}\right)$ from lattice QCD, Nucl. Phys. A931 (2014) 851-855, [1408.6305].

[12] S. Borsanyi, G. Endrodi, Z. Fodor, S. D. Katz, S. Krieg, C. Ratti et al., QCD equation of state at nonzero chemical potential: continuum results with physical quark masses at order $\mathrm{mu}^{2}$, JHEP 08 (2012) 053, [1204.6710].

[13] A. Bazavov et al., The QCD Equation of State to $\mathscr{O}\left(\mu_{B}^{6}\right)$ from Lattice $Q C D$, Phys. Rev. D95 (2017) 054504, [1701.04325].

[14] H. Akaike, Information theory and an extension of the maximum likelihood principle. Springer, 1992.

[15] P. Alba, W. Alberico, R. Bellwied, M. Bluhm, V. Mantovani Sarti, M. Nahrgang et al., Freeze-out conditions from net-proton and net-charge fluctuations at RHIC, Phys. Lett. B738 (2014) 305-310, [1403.4903].

[16] STAR collaboration, L. Adamczyk et al., Energy Dependence of Moments of Net-proton Multiplicity Distributions at RHIC, Phys. Rev. Lett. 112 (2014) 032302, [1309. 5681].

[17] S. Durr et al., Ab-Initio Determination of Light Hadron Masses, Science 322 (2008) 1224-1227, [0906.3599].

[18] Jülich Supercomputing Centre, Juqueen: Ibm blue gene/q supercomputer system at the jülich supercomputing centre, Journal of large-scale research facilities A1 (2015) 1. 